\section{Modified visual field test for ptosis surgery (Leicester Peripheral Field Test)}

SF Ho, A Morawski, R Sampath and J Burns

\begin{abstract}
Introduction There is lack of consensus among Primary Health Care Trusts (PCTs) and health insurers on how to reimburse ptosis surgery and upper lid blepharoplasty, as these procedures can be regarded as cosmetic. Standardised photographs are expensive and difficult to achieve, whilst the routine 24-2 visual field lacks the range to detect visually significant superior field defects. Aim To introduce a modified visual field designed to assess the functional disability associated with ptosis and dermatochalasis and to demonstrate the effectiveness of surgery in improving the visual field. Methods Patients who had surgery for ptosis or dermatochalasis between January 2006 and December 2009 were prospectively invited to perform a modified visual field test pre- and post-operatively.
\end{abstract}

Results In total, 97 patients amounting to 194 eyes were included in the study. Ninety five eyes had aponeurotic repair with or without blepharoplasty and 77 eyes had blepharoplasty alone. This modified test has a sensitivity of $\mathbf{9 8 . 8 \%}$ of detecting ptosis. For patients who underwent ptosis surgery with or without blepharoplasty, $84.2 \%$ recorded an improvement in points seen with the test and $81 \%$ recorded an improvement in visual field height. For those who had blepharoplasty alone, $\mathbf{9 0 . 9 \%}$ recorded an improvement in points seen in the modified visual field test and $80.6 \%$ had improvement in visual field height.

Conclusion Our modified visual field assessment is a quick and easy way to assess patient disability associated with ptosis and dermatochalasis. Surgery improves the demonstrated defect, confirming that ptosis and dermatochalasis can be considered a functional rather than cosmetic issue. Eye (2011) 25, 365-369; doi:10.1038/eye.2010.210; published online 21 January 2011
Keywords: modified visual field test; ptosis; dermatochalasis

\section{Introduction}

Funding or reimbursement of ptosis surgery and blepharoplasty within the healthcare setting has long been a contentious issue. ${ }^{1}$ Various arbitrary guidelines have been issued by medical insurers and Primary Health Care Trusts (PCTs). ${ }^{2-6}$ The lack of consistency and clarity of these guidelines has created a significant amount of confusion, causing anxiety for patients and raising questions of a postcode lottery. The requirement from some PCT for an individual case review for each procedure has also created a significant workload for those involved in the decision making process. For example, for one particular medical insurer, the criteria to prove that ptosis is a functional disability includes provision of a full-face photograph demonstrating that the upper lid droops over the pupillary aperture to a degree consistent with the abnormal visual field. For blepharoplasty, it is for photographs demonstrating dermatochalasis and a visual field showing a defect to at least $30^{\circ}$ above the visual axis and which is significantly improved or restored when the lid is taped. ${ }^{2}$ Other insurer's guidance reads 'blepharoplasty will be commissioned for eyelid ptosis and/or excess skin of the upper eyelid, which causes obscured vision'. ${ }^{4}$

In order for a photograph to be considered, it is stipulated that the reflected light from the flash used to illuminate the subject must be in the central area of the patient's face. However, this is difficult to achieve without a trained photographer in a standardised setting, which has significant cost implications. The validity of the results may also be affected by the variability of the height of the eyelid over time,
Department of

Ophthalmology, Leicester Royal Infirmary, Leicester LE1 5WW, UK.

Correspondence: SF Ho, Ophthalmology Department, Queen Alexandra Hospital, Cosham PO6 3LY, UK.

Tel: 0239228 6931; E-mail: s_ho2@yahoo.com

Received: 26 May 2010 Accepted in revised form: 14 November 2010 Published online: 21 January 2011

This project has been presented as a poster in British Oculoplastic Surgery Society (BOPSS) meeting, Edinburgh in June 2010. It was presented as an oral presentation in the European Society of Ophthalmic Plastic and Reconstructive Surgery (ESOPRS) meeting, Munich in September 2010.

It was also being presented in Midlands

Ophthalmological Society in September 2010. 
chin position, difficulty in fixation, frontalis over action and even the patient's mood and expression.

Whilst the visual field has been used in some ophthalmic units to prove the presence of ptosis, there is so far no standardized visual field designed for this purpose. In the United Kingdom, most centres use either Goldmann visual field or Humphrey 24-2 as is used for glaucomatous patients. Although Goldmann test is sensitive and comprehensive, it is also time consuming, operator dependent and less specific, as some patients can predict the next point. The routine Humphrey 24-2 only tests a limited superior $24^{\circ}$ field, which is far less than that used by most people for normal activities of daily living. Patients with ptosis and dermatochalasis often complain of a superior visual field defect that is not detectable with 24-2 testing, but which is influencing their visual function and confidence for activities such as driving and crossing the road, or their occupational requirements such as in engineering. This is supported by Small et al's study showing that $97 \%$ of patients with a marginal reflex distance of $2 \mathrm{~mm}$ or less had upper visual field restriction of $30^{\circ}$ or less. ${ }^{7}$ In the United States, our American colleagues use Goldmann or automated static perimetry, which is either a $10 \mathrm{~dB}$ threshold (Humphrey analyzer) or a $7 \mathrm{~dB}$ threshold (Octopus analyzer). ${ }^{8}$

Although the latter is accurate, it is time consuming and subject to a huge learning curve with high false positives and negatives. Meanwhile, various custom Humphrey field tests so far reported in the literature, which test more points superiorly have limitations such as not testing points in inferior field, ${ }^{9,10}$ which may induce bias, and threshold static testing, ${ }^{8,11}$ which is time consuming.

We therefore designed a modified visual field test specifically to assess ptosis and dermatochalasis. We aim to use this new assessment tool to demonstrate the functional disability associated with these conditions and the effectiveness of surgery in improving the superior visual field.

\section{Subjects and methods}

This was a prospective study performed on patients who were referred to the Leicester Royal Infirmary

Ophthalmology Department with ptosis or dermatochalasis between January 2006 and December 2009. Patients who were referred for surgery were requested to perform a modified Humphrey visual field test. The inclusion criteria included upper lid malposition of various causes, such as dermatochalasis, levator aponeurotic dehiscence, and congenital ptosis. However, those with brow ptosis, asymmetry with unilateral anophthalmic socket or other known ocular pathology associated with a visual field defect, such as glaucoma, optic neuropathy, diabetic retinopathy (eg, previous panretinal photocoagulation) or other neurological problems were excluded. Twenty healthy individuals (40 eyes) with an average age of 51 were also tested; only three eyes in three separate patients showed just three contiguous absolute missed points. We have defined ptosis as a margin reflex distance (MRD) of $2 \mathrm{~mm}$ or less. ${ }^{7}$

\section{Construction of the test}

The modified visual field test was jointly designed by two of our authors, AM and JB. It has been named as Leicester Peripheral Field Test. It is an age corrected screening test with a three zone strategy (Figure 1). Two grids were created on either side of the $x$ axis.

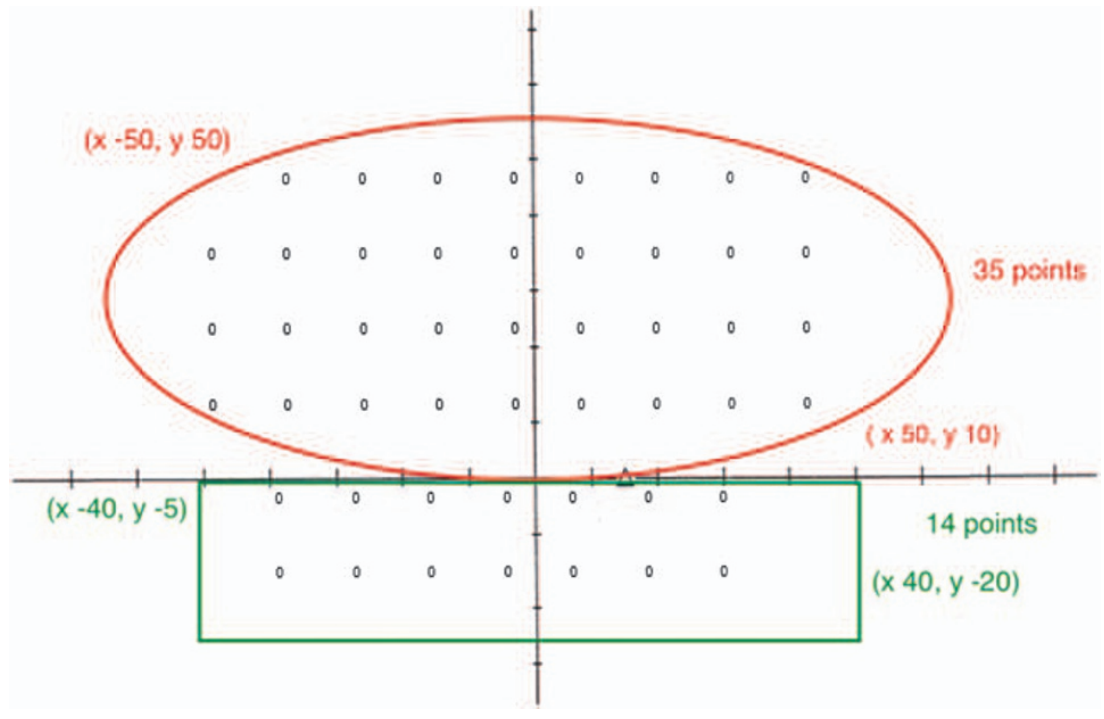

Figure 1 Modified visual field text. 
Grid A (above the $x$ axis) has the following co-ordinates:

Top left corner: $x,-50 ; y, 50$

Bottom right corner: $x, 50 ; y, 10$

Grid B (below the $x$ axis) has the following co-ordinates:

Top left corner: $x,-40 ; y,-5$

Bottom right corner $x, 40 ; y,-20$

Further information about the setting-up of the test can be obtained via e-mail at joyce.burns@uhl-tr.nhs.uk

Thirty five points are tested in the superior field whilst 14 points are tested in the inferior field. In total, a maximum of $48^{\circ}$ will be tested in the superior visual field. The inferior field test serves as a reference, but is not used in the analysis.

Patients were seated $33 \mathrm{~cm}$ from the target, without corrective lenses, and the centre of fixation is shifted $15^{\circ}$ inferiorly to allow for maximum superior field-testing. A defect in the visual field is defined as absolute when the stimulus is not being seen at $0 \mathrm{~dB}$. A relative defect identifies a stimulus not seen at $6 \mathrm{~dB}$ above the reference threshold, but seen at an intensity of $0 \mathrm{~dB}$. There was a tendency for ptosis patients to have a more central defect as opposed to the more temporal defect seen in dermatochalasis, but this was not found to be statistically significant. The height of the defect is defined as the lowest point in degrees where there are three horizontal contiguous absolute defects.

\section{Results}

In total, 97 patients with 194 eyes were included in the study. Thirty six were male and 61 were female. The age range was 20-87 with median of 67 .

The extent of visual field defect associated with ptosis, dermatochalasis or a combination ranged between 0 to 35 points missed with a median of 19 (mean 19, SD 9.32). Post-operatively the number of points missed ranged from 0 to 33 , but the median improved to 4 (mean 7, SD 7.62). The height of visual field before surgery ranged between 0 to $48^{\circ}$ with a median of $12^{\circ}$ (mean 18 , SD 12.8).

Post-operatively the height of the field ranged from 12 to $48^{\circ}$ with an improved median of $36^{\circ}$ (mean 37.87, SD 9.14). The time taken to perform the test pre-operatively was $2.20-6.47 \mathrm{~min}$, with a median of $4.18 \mathrm{~min}$ (mean $4.04 \mathrm{~min}, \mathrm{SD} 0.58 \mathrm{~min}$ ). Time taken to perform the test post-operatively ranged from 2.20 to $6.17 \mathrm{~min}$ with the median reduced to $3.27 \mathrm{~min}$ (mean $3.29 \mathrm{~min}$, SD $0.53 \mathrm{~min}$ ).

A binary statistical calculation was performed to identify the relationship between the presence of a visual field defect and ptosis. In total, 84 out of 85 eyes with ptosis had a visual field defect, which lead to a sensitivity of $98.8 \%$. (95\% Confidence interval: $93.6-100 \%$ )
Meanwhile, 37 eyes out of 40 eyes who did not have ptosis or dermatochalasis did not have a visual field defect (specificity of $92.5 \%$ with $95 \%$ confidence interval of $79.6-98.4 \%$ ). The test therefore has a positive predictive value of $96.6 \%$, negative predictive value of $97.4 \%$ and false positive rate of $7.5 \%$ (Table 1 ).

After ptosis surgery with or without blepharoplasty, there was a change in the number of points seen ranging from -9 to +35 (median 13, mean 12.9, SD 9.5). In total, 80 out of 95 eyes (84.2\%) improved, 5 eyes (5.2\%) were unchanged and 10 eyes $(10.5 \%)$ had deterioration in the number of points seen (Figures 2 and 3). In 20 eyes (21\%) the number of points seen improved by more than 20 points. In a further 37 eyes $(39 \%)$ the improvement in points seen was between 11 and 20, and in the remaining 23 eyes $(24.2 \%)$ there was an improvement in 10 or less points. For the 10 eyes who saw fewer points after ptosis surgery, 7 had deterioration by only 5 points or less compared with before the operation (Figure 3).

Table 1 Sensitivity and specificity of the visual field test in identifying ptosis

\begin{tabular}{lccr}
\hline & \multicolumn{2}{c}{ Ptosis $(+1-$ dermatochalasis $)$} & Total \\
\cline { 2 - 3 } & Present & Absent & \\
\hline Visual field defect & 84 & 3 & 87 \\
No visual field defect & 1 & 37 & 38 \\
Total & 85 & 40 & 125 \\
\hline
\end{tabular}

Sensitivity: $84 / 85=98.8 \%$ (95\% confidence interval: $93.6-100 \%)$ Specificity: $37 / 40=92.5 \%$ (95\% confidence interval: $79.6-98.4 \%$ ) Positive predictive value: $84 / 87=96.6 \%$ (95\% confidence interval: 90.2-99.2\%)

Negative predictive value: $37 / 38=97.4 \% \quad(95 \%$ confidence interval: 86.2-100\%)

False positive value: $3 / 40=7.5 \%$

\section{Comparison of preop and post-op points missed after ptosis surgery +1 - blepharoplasty}

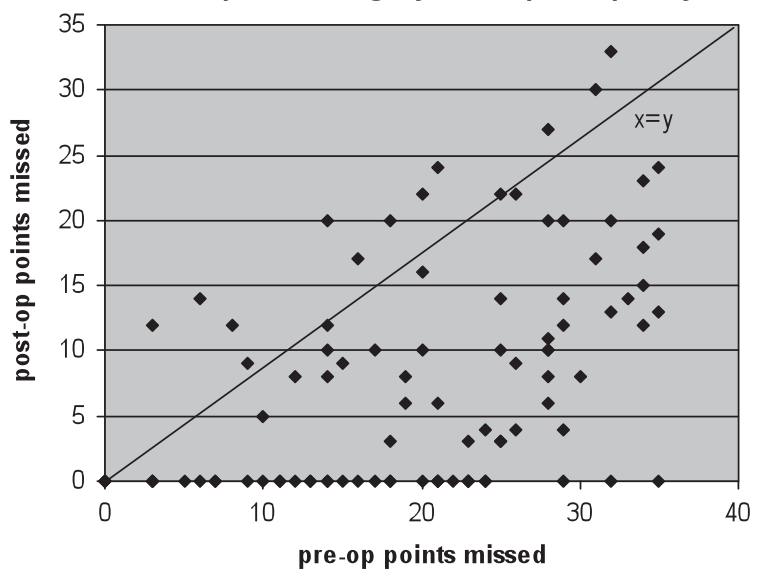

Figure 2 Comparison of pre-op and post-op points missed after ptosis surgery $+/$-blepharoplasty. 


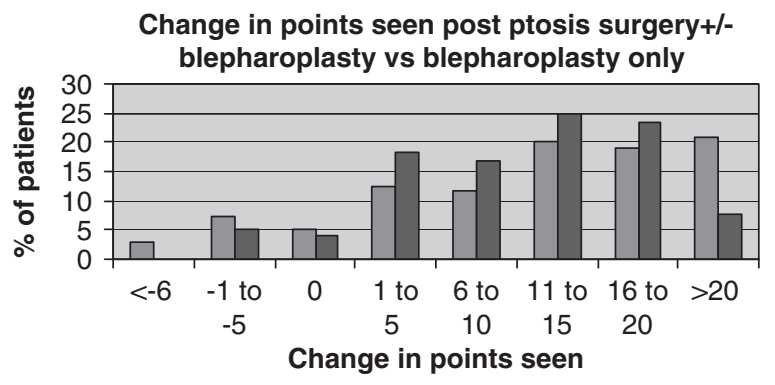

$\square$ post ptosis surgery $+/-\quad \square$ post blepharoplasty blepharoplasty

Figure 3 Change in points seen after ptosis surgeries $+/-$ blepharoplasty $v s$ blepharoplasty only.

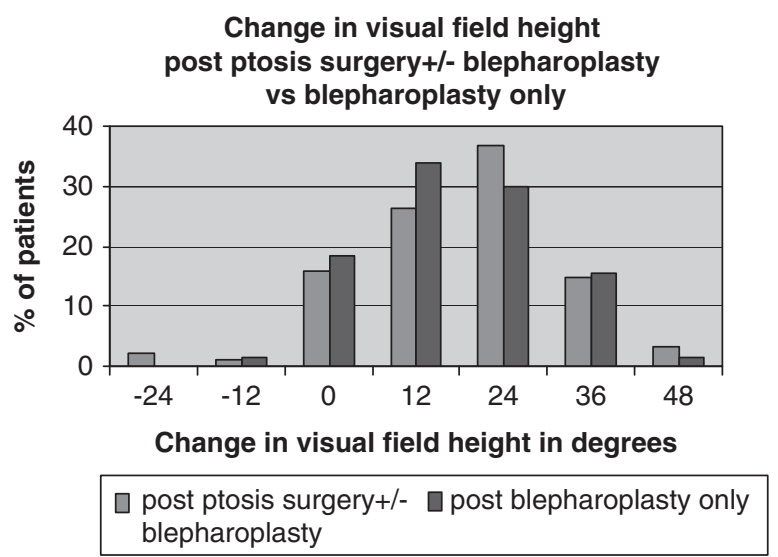

Figure 4 Change in visual field height post-ptosis surgeries with and without blepharoplasty vs blepharoplasty only.

Regarding visual field height, 75 eyes (81\%) had improvement in visual field height post-ptosis surgery with or without blepharoplasty. Fifteen eyes (15.8\%) had no improvement, whilst 3 eyes (3.1\%) had a reduction (within $24^{\circ}$ ) in visual field height post-ptosis surgery (Figure 4).

Dermatochalasis constitutes a special group as blepharoplasty has long been considered as cosmetic surgery. There were 39 patients who had blepharoplasty with 38 bilateral and one unilateral, which accounted for 77 eyes in total. The number of points missed before blepharoplasty ranged from 2 to 35 (median 17, mean 17.44, SD 8.72), and the height of superior visual field in this group ranged from 0 to $48^{\circ}$ (median 24, mean 20.26, SD 12.2). After blepharoplasty there was a change in the number of points seen ranging from -4 to +29 (median 12 , mean 10.83 , SD 7.42). Seventy eyes (90.9\%) had an improvement in points seen post blepharoplasty while 3 (3.5\%) were unchanged and $4(5.2 \%)$ saw up to five points fewer than pre-op (Figure 3). Sixty two eyes (80.6\%) demonstrated an improvement in visual field height after surgery (Figure 4).

\section{Discussion}

In the United Kingdom, the increased tightening of the health budget has brought the issue of 'cosmetic' procedures funded by the NHS into sharper focus. Although visual field tests have been used to demonstrate objectively deficits associated with ptosis or dermatochalasis, ${ }^{9-15}$ the previously used techniques proved to be time consuming, ${ }^{10,11}$ operator dependent ${ }^{8}$ or inadequate.

We have chosen the Humphrey visual field analyzer as it is available in most ophthalmic units in the United Kingdom and therefore has minimal impact on the cost of assessing patients once referred. The greater number of points being tested in the superior visual field provides improved sensitivity in detecting superior visual loss associated with upper lid malposition. Points in the inferior field reduce bias by avoiding preferential looking at the superior visual field. The inferior visual field also serves as a useful guide to the technician that the patient understands the test well enough to perform reliably. Shifting the central fixation $15^{\circ}$ inferiorly allows the superior field to be tested up to $48^{\circ}$ within the existing Humphrey frame. The slightly down gaze position also counteracts the involuntary frontalis over action in some ptotic patients and therefore reduces this bias. The significance of down gaze ptosis causing difficulty in reading and fine work has been emphasized in recent literature., ${ }^{9,16,17}$

The automated nature of this modified test means it is not operator dependent and could be performed by the optician who made the referral. Testing specifically the superior field also means a shorter testing time, as demonstrated by our results that the majority of tests can be done within $5 \mathrm{~min}$. This is significantly faster than the custom static full-threshold $60^{\circ}$ test strategy as suggested by Meyer et $a l^{11}$ and has advantages for patients with ptosis as they are often elderly and may have other health co morbidities causing difficulty with prolonged concentration.

We also chose not to perform taped and untaped measurements as published data does not support the idea that improved fields with taping or manual elevation of the eyelid is predictive of improved function after surgery. ${ }^{18}$ Many experts also feel that manually elevating the upper eyelid will underestimate the actual visual field improvement after ptosis surgery and simply adds to the time and expense of the pre-operative assessment. ${ }^{6}$

The validity of this test was evidenced by the high sensitivity and positive predictive value, which were 98.8 and $96.6 \%$, respectively. We therefore believe this will be a very useful initial test to prove a functional deficit in upper lid malposition for the consideration of reimbursement after surgery. 
This large study clearly demonstrates that ptosis and dermatochalasis may be associated with superior visual field defects and is comparable with other studies. ${ }^{9-13}$ We have also shown that ptosis surgery and blepharoplasty will improve the visual field as evidenced by the increase in the height as well as number of points seen in the visual field. Although it is hard to be dictatic about the extent of the defect before patients are considered eligible for surgery, we propose that any three contiguous points missed up to $48^{\circ}$ should be considered a significant defect and that those patients will benefit from intervention.

However, we acknowledge that although our modified visual field is a good assessment tool for ptosis, it has limitations. As this test is a screening test rather than a threshold test, it may not be able to identify a visual field defect associated with more subtle degrees of ptosis. ${ }^{11}$ Therefore, the few patients complaining of a reduced visual field who have no measured defect could be offered ancillary tests, such as photographs, a threshold visual field test or a quality of life questionnaire. Other patients with obvious ptosis who struggle to sit at the analyzer or maintain adequate concentration should not be denied surgery on the basis of this test alone.

We agree with Meyer ${ }^{6}$ that more study on this topic is needed so that third party insurers and Primary Care Trusts ( PCT) can develop uniform specific criteria for functional blepharoptosis, and offer this work as a move towards that goal.

\section{Conclusion}

We present here a modified visual field test (Leicester Peripheral Field Test), which is valid, quick and easy to perform. It demonstrates the visual field defects associated with both ptosis and dermatochalasis, and illustrates the improvement in visual function after corrective surgery. We therefore believe it to be a useful assessment tool providing evidence for reimbursement by medical insurers or PCT.

\section{Summary}

\section{What was known before}

- Goldmann visual field test and Humphrey field 24-2 is being used to establish visual field loss in ptosis and dermatochalasis.

\section{What this study adds}

- We introduce a modified visual field test, which is faster, more sensitive, and valid in demonstrating visual field defect.

\section{Conflict of interest}

The authors declare no conflict of interest.

\section{Acknowledgements}

We are grateful for the help of Mr Bernie Higgins from Portsmouth University for his statistical advice. We are also grateful for the help of Mr Jonathan Bhargava and Mrs Katya Tambe for their initial work on this project.

\section{References}

1 Anderson RJ, Holds JB. Does anyone know how to differentiate a 'functional' defect from a cosmetic one? Arch Ophthalmol 1990; 108: 1685-1686.

2 United Healthcare Oxford. Repair of Ptosis, Policy number : SURGERY 018.6 T2.

3 Nottinghamshire County Teaching Primary Care Trust. Commissioning policy cosmetic plastic surgery procedures 2008.

4 Norcom Commissioning Policy Specialist Plastic Surgery Procedures 2007.

5 South Staffordshire Primary Care Trust. Commissioning policy and Referral guidelines for provision of Aesthetic Surgery for NHS Patients. Policy effective from 1 September 2008.

6 Meyer DR. Functional eyelid surgery. Ophthal Plast Reconstr Surg 1997; 13: 77-80.

7 Small R, Sebates NR, Burrows D. The measurement and definition of ptosis. Ophthal Plast Reconstr Surg 1989; 5(3): 171-175.

8 American Medical Association. The Visual System. In: Engelberg AL (ed). Guide to the evaluation of permanent impairment 3rd Ed (rev). American Medical Association: Chicago, 1990, pp 153-164.

9 Patipa M. Visual field loss in primary gaze and reading gaze due to acquired blepharoptosis and visual field improvement following ptosis surgery. Arch Ophthalmol 1992; 110: 63-67.

10 Hacker HD, Hollsten DA. Investigation of automated perimetry in the evaluation of patients for upper lid blepharoplasty. Ophthal Plast Reconstr Surg 1992; 8: 250-255.

11 Meyer DR, Stern JH, Jarvis JM, Liniger LL. Evaluating the visual field effects of blepharoptosis using automated static perimetry. Ophthalmology 1993; 100: 651-659.

12 Cahill KV, Burns JA, Weber PA. The effect of blepharoptosis on the field of vision. Ophthalmic Plast Reconstr Surg 1987; 3: 121-127.

13 Meyer DR, Linberg JV, Powell SR, Odom JV. Quantitating the superior visual field loss associated with ptosis. Arch Ophthalmol 1989; 107: 840-843.

14 Small RG. Discussion of above reference. Ophthalmology 1993; 100: 658-659.

15 Small RG. Functional vs cosmetic ophthalmologic defects. Arch Ophthalmol 1994; 109: 1194-1195.

16 Dryden RM, Kahanic DA. Worsening of blepharoptosis in down gaze. Ophthal Plast Reconstr Surg 1992; 8: 126-129.

17 Wojno TH. Down gaze ptosis. Ophthal Plast Reconstr Surg 1993; 9: 83-89.

18 Federici T, Meyer DR, Lininger LL. Correlation of the vision-related functional impairment associated with blepharoptosis and the impact of blepharoptosis surgery. Ophthalmology 1999; 106: 1705-1712. 